\title{
Investigation of the effect of short-term change in dietary magnesium intake in asthma
}

\author{
J. Hill*, A. Micklewright**, S. Lewis*, J. Britton*
}

Investigation of the effect of short-term change in dietary magnesium intake in asthma. J. Hill, A. Micklewright, S. Lewis, J. Britton. C ERS Journals Ltd 1997.

ABSTRACT: Epidemiological evidence suggests that a low dietary intake of magnesium is associated with impaired lung function, bronchial hyperreactivity and wheezing. This study was designed to investigate whether short-term alterations of dietary magnesium intake have an effect on the clinical control of asthma.

In a randomized, double-blind, placebo-controlled, cross-over study, 17 asthmatic subjects adhered to a low magnesium diet for two periods of 3 weeks, preceded and separated by a 1 week run-in/wash-out, in which they took either placebo or magnesium (400 $\mathrm{mg} \cdot \mathrm{day}^{-1}$ ) tablet supplementation. Forced expiratory volume in one second (FEV1) and the provocative dose of methacholine required to cause a $20 \%$ fall in FEV1 from baseline $\left(\mathrm{PD}_{\left.20, \mathrm{FEV}_{1}\right)}\right.$ were measured at the beginning and end of each treatment period, and variation in peak expiratory flow (PEF) rate, bronchodilator use and symptom scores recorded throughout.

Asthma symptom scores were significantly lower during the magnesium treatment period, the median ( $95 \%$ confidence interval) difference from placebo being $3.8(0.5-7.0)$ symptom points per 7 days $(p=0.02)$. However, there was no significant improvement in FEV1, PD20,FEV1, log amplitude percentage mean PEF variation or bronchodilator use during magnesium supplementation.

A high magnesium intake was associated with improvement in symptom scores, though not in objective measures of airflow or airway reactivity, in these stable asthmatic subjects.

Eur Respir J 1997; 10: 2225-2229.

Magnesium is a dietary cation with several actions of potential benefit to the asthmatic airway, including: inhibition of contraction of vascular [1] and bronchial [2, $3]$ smooth muscle in vitro; inhibition of release of acetylcholine from cholinergic nerve terminals [4] and of histamine from mast cells [5]; and promotion of nitric oxide [6] and prostacyclin generation [7]. Intravenous [8-13] and inhaled [14-16] magnesium has been shown to be beneficial in several studies, both of acute and stable asthma, although not all studies have confirmed this [17-21]. We have recently reported epidemiological evidence that a lower dietary magnesium intake in the general population is associated with impaired lung function, bronchial hyperreactivity and increased risk of wheezing [22]. Magnesium may, therefore, have a role in the aetiology and therapy of asthma. This randomized, double-blind, placebo-controlled, cross-over study was designed to determine whether short-term alteration of dietary magnesium intake influences the control of stable asthma.

\section{Materials and methods}

\section{Subjects}

Twenty nonsmoking asthmatic subjects aged 25-60 yrs were recruited to take part in this study. All had been shown to increase their forced expiratory volume
*Division of Respiratory Medicine, and **Dept of Dietetics, City Hospital, Nottingham, UK.

Correspondence: J. Britton

Division of Respiratory Medicine

University of Nottingham

City Hospital

Hucknall Road

Nottingham NG5 1PB

UK

Keywords: Asthma

diet

magnesium

Received: January 71997

Accepted after revision June 281997

This study was funded by a grant from the Astra Foundation, UK. in one second (FEV1) by at least $15 \%$ after $200 \mu \mathrm{g}$ inhaled salbutamol and to have a provocative dose of methacholine required to cause a $20 \%$ fall in FEV1 from baseline (PD20,FEV 1 ) of less than $12.24 \mu \mathrm{mol}$ at a screening visit or within 3 months of commencement of the study, and all were symptomatic to the point of requiring inhaled beta-agonist therapy at least once per $24 \mathrm{~h}$. Subjects were included if they were taking inhaled corticosteroids or oral methylxanthines but were instructed not to change the dose of these drugs during the study. Subjects were asked to withhold inhaled betaagonists, sodium cromoglycate and caffeine-containing beverages for $6 \mathrm{~h}$ prior to each visit, and oral methylxanthines for $12 \mathrm{~h}$ prior to each visit. None was taking any treatments likely to affect magnesium absorption or excretion, including diuretics, digoxin, calcium-containing medications or excess alcohol. All subjects gave written consent. The study was approved by the City Hospital Ethics Committee. Exemption from product licence and clinical trial certificate requirements for the use of magnesium in the study was granted by the UK Medicines Control Agency.

\section{Study design}

The study used a randomized, cross-over, double-blind, placebo-controlled design in which all subjects were 
asked to adhere to a low magnesium diet, and then received either placebo or magnesium supplementation. During a 7 day run-in period eating their normal diet, subjects used electronic scales (Salter, Kent, UK) to weigh and record all food and beverage consumption for 1 week in a food diary. Subjects recorded twice daily peak expiratory flows (PEF) measured as the best of three manoeuvres using a Mini-Wright meter (Clement Clarke International Ltd, UK), twice daily asthma symptom scores of wheeze, shortness of breath and sleep quality, each on a scale from 0 to 4 points, and the number of bronchodilator doses used each day on a personal diary card.

Food diaries were then analysed to determine the mean daily magnesium intake over that period using the Comp-eat software programme, version 4.0 (Nutrition System, London, UK), and at the second visit subjects were advised on minor dietary alterations which would reduce, if necessary, their dietary magnesium intake to 100-200 mg.day ${ }^{-1}$. Subjects were asked to adhere to these dietary restrictions for two periods of 3 weeks, during which they received placebo or magnesium tablets according to a randomized, double-blind protocol. Magnesium was replaced in the form of magnesium amino acid chelate tablets (Lamberts Healthcare, Luton, UK) each containing $200 \mathrm{mg}$ (or $8.2 \mathrm{mmol}$ ) of elemental magnesium, and each subject received two tablets (16.4 mmol) per day during the magnesium supplementation arm of the study. Measurements of 7 day magnesium intake were repeated, by the same method as in the run-in period, during the middle 7 days of each treatment period. There was a 1 week wash-out period between the two trial periods, during which dietary restrictions were lifted but subjects continued to record peak flows, symptom scores and bronchodilator use.

Measurements of FEV1 and PD20,FEV1 to methacholine were made, and a venous blood sample taken for serum and leucocyte magnesium estimation at the beginning and end of each of the two 3 week trial periods. Subjects collected urine for $48 \mathrm{~h}$ for estimation of magnesium excretion prior to the beginning and end of each treatment period. Leucocyte magnesium concentrations were measured by absorption flame spectrophotometry after leucocyte separation according to the method described by ELIN and Hosseini [23].

\section{Methods}

FEV1 was measured using a dry bellows spirometer (Vitalograph, Buckingham, UK) after 15 min rest in the seated position, and taking the best of two attempts within $100 \mathrm{~mL}$. PD20,FEV 1 to methacholine was measured by the method of YAN et al. [24]. Methacholine aerosols were delivered from a series of De Vilbiss handheld nebulizers, delivering $10-11 \mu \mathrm{L} \cdot$ breath $^{-1}$. Subjects inhaled doubling doses of methacholine (from 0.048 to $12.24 \mu \mathrm{mol}$ ) during rapid inspiration from functional residual capacity (FRC) to total lung capacity (TLC). Subjects were asked to hold their breath for $3 \mathrm{~s}$ after each inhalation, and then to exhale slowly for $3 \mathrm{~s}$. FEV1 was measured $1 \mathrm{~min}$ after each inhalation. The challenge was discontinued when the FEV1 had fallen by $20 \%$ or more from the baseline value, and
PD20,FEV1 was calculated by linear interpolation on a log dose-response plot. PD20,FEV1 measures by this method have been shown to be of comparable repeatability with other more complex techniques [25]. Censored PD20,FEV1 values of $12.24 \mu \mathrm{mol}$ were assigned on occasions when PD20,FEV1 rose above the upper dose limit of $12.25 \mu \mathrm{mol}$ during the course of the study.

\section{Analysis}

Period and carry-over effects were calculated according to the method described by Hills and ARMitage [26]. Amplitude percentage mean PEF was calculated as the difference between evening and morning PEF divided by the mean of the two measurements, averaged over the run-in/wash-out period, and over the last 7 days of each treatment period. FEV1 and PD20,FEV1 at the end of, and amplitude percentage mean PEF during the run-in and wash-out periods were taken as baseline levels for the first and second treatment periods, respectively. Amplitude percentage mean and PD20,FEV1 were $\log _{10}$ transformed in all analyses. Change from baseline in FEV1, $\log _{10}$ PD20,FEV1 and log amplitude percentage mean peak flow were compared between each of the two 3 week periods by analysis of variance (ANOVA) within subjects using the Statistical Package for the Social Sciences (SPSS)-PC version 4.1. Cumulative symptom scores and cumulative number of puffs of betaagonist used for the 7 day run-in and wash-out periods and for the last 7 days of each treatment period were calculated, and the change in the scores from run-in (first treatment period) or wash-out (second treatment period) compared using Wilcoxon Rank tests for nonparametric data using the statistical software package Minitab (Edinburgh University Computing Service and Minitab Inc., PA, USA). Serum and leucocyte magnesium and $48 \mathrm{~h}$ magnesium excretion at the end of each treatment period were compared by Student's t-test for paired data.

\section{Power}

The primary end-point used in designing the study was FEV1, and a $150 \mathrm{~mL}$ difference in FEV1 was arbitrarily chosen to be a relevant outcome. Using published evidence that the within-subject standard deviation for repeated measures of FEV1 is of the order of 150 $\mathrm{mL}[27,28]$, the study was designed to provide approximately $90 \%$ power at the $5 \%$ significance level for a change of this magnitude.

\section{Results}

Of the 20 subjects recruited to take part in the study, one withdrew after 4 days in the first treatment period (placebo arm) because of concerns that the study tablets were affecting his fitness, and through misunderstanding of the protocol requirements, and two subjects did not complete diary cards during the wash-out period. Complete data on FEV1, PD20,FEV1, symptom scores, amplitude percentage mean peak flow and bronchodilator 
Table 1. - Mean FEV1, $\log _{10}$ PD20,FEV1 to methacholine, log amplitude percentage mean PEF, and cumulative symptom scores and bronchodilator use for the last 7 days of run-in, wash-out and placebo and magnesium treatment periods

\begin{tabular}{lcccccccc}
\hline Measure & $\begin{array}{c}\text { Beginning of } \\
\text { placebo }\end{array}$ & $\begin{array}{c}\text { End of } \\
\text { placebo }\end{array}$ & $\begin{array}{c}\text { Beginning of } \\
\text { magnesium }\end{array}$ & $\begin{array}{c}\text { End of } \\
\text { magnesium }\end{array}$ & $\begin{array}{c}\text { Mean } \\
\text { difference** }\end{array}$ & 95\% CI & p-value & Test \\
\hline FEV1 L & 2.548 & 2.483 & 2.486 & 2.494 & 0.072 & -0.083 to 0.227 & 0.29 & ANOVA \\
Log $_{10}$ PD20,FEV1 & 0.087 & 0.203 & 0.069 & -0.120 & -0.30 & -0.63 to 0.02 & 0.09 & ANOVA \\
Log $_{10}$ amplitude $\%$ & 0.81 & 0.78 & 0.83 & 0.81 & 0.012 & -0.15 to 0.17 & 0.79 & ANOVA \\
mean PEF & 5 & 6 & 6 & 2 & -3.8 & -7.0 to -0.5 & 0.02 & Wilcoxon \\
$\begin{array}{l}\text { Symptom } \\
\text { scores*\# }\end{array}$ & $(0-24)$ & $(0-25)$ & $(0-17)$ & $(0-16)$ & -6.0 & -10.5 to 1 & 0.051 Wilcoxon \\
$\begin{array}{l}\text { Bronchodilator } \\
\text { use** }\end{array}$ & 20 & 14 & 24 & 11 & & & & \\
\hline
\end{tabular}

*: median, and range in parenthesis; **: mean differences between change during magnesium and change during placebo treat-

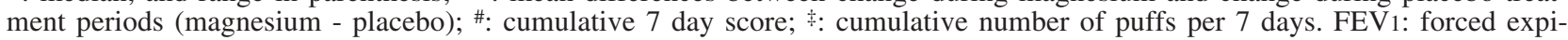
ratory volume in one second; PD20,FEV1: provocative concentration of methacholine causing a 20\% fall in FEV1 from baseline; PEF: peak expiratory flow; 95\% CI: 95\% confidence interval; ANOVA: analysis of variance.

use are, therefore, available on 17 subjects (10 males and 7 females; mean age (range) 44.8 (22-59) yrs) (table 1). Three PD20,FEV1 values (4.4\% of total number of measurements) were censored at $12.24 \mu \mathrm{mol}$. At the end of the run-in period: mean (SD) FEV1 was 2.47 (0.56) $\mathrm{L}$; mean (range) $\mathrm{FEV} 1 \%$ predicted was 77 (55-112)\%; mean (SD) $\log _{10}$ PD20,FEV1 was 0.16 (0.57); mean (SD) $\log _{10}$ amplitude percentage mean PEF was 0.84 (0.35); median (range) cumulative 7 day symptom score was 7 (0-24) symptom points; and median (range) cumulative bronchodilator use was 24 (0-58) doses week $^{-1}$. Sixteen subjects were receiving regular inhaled corticosteroids, two subjects were taking sodium cromoglycate and one was taking an oral methylxanthine. Mean magnesium intake during the run-in period was 294 (range 178-565) $\mathrm{mg} \cdot \mathrm{day}^{-1}$, and baseline magnesium intake was unrelated to baseline physiology or symptoms. During the middle week of the placebo and magnesium treatment periods, mean magnesium intake was 210 and $211 \mathrm{mg} \cdot \mathrm{day}^{-1}$, respectively, and the subjects were, therefore, consuming an average of approximately 210 and $610 \mathrm{mg} \cdot \mathrm{day}^{-1}$ (or 8.2 and 24.6 mmol.day ${ }^{-1}$ ) magnesium during the low and high magnesium treatment periods, respectively. There were no appreciable adverse effects reported during either treatment period.

There was no significant effect of treatment on change

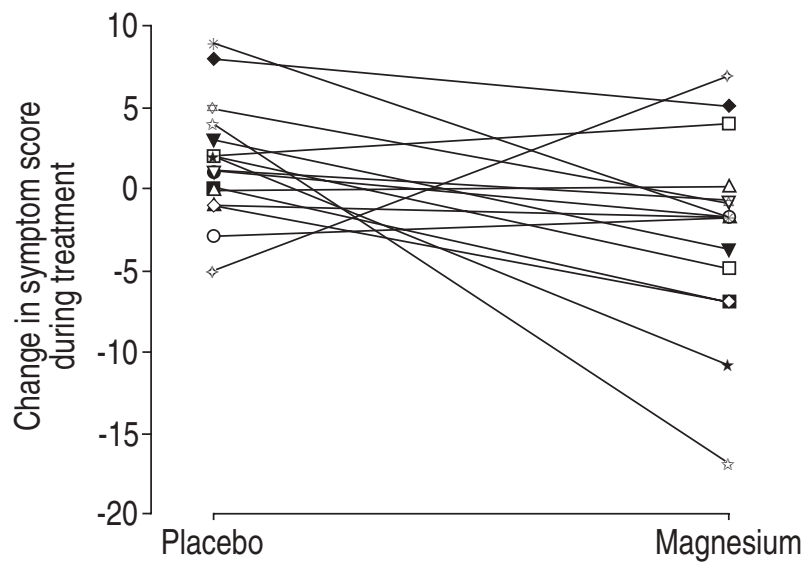

Fig. 1. - Change in 7 day cumulative symptom scores with placebo or magnesium supplementation during adherence to a low magnesium diet in 17 asthmatic subjects. Each subject is represented by a different symbol. in FEV1 (mean difference (95\% confidence interval (CI)) 0.072 (-0.083-0.227) L higher with magnesium; $\mathrm{p}=0.29$ ), PD20,FEV1 (mean difference (95\% CI) 0.30 $(-0.02-0.63)$ higher $\log _{10}$ PD20,FEV1 with placebo; $p=$ 0.09 ), or $\log _{10}$ amplitude percentage mean PEF (mean difference (95\% CI) $0.012(-0.15-0.17)$ higher with magnesium; $p=0.79)$. There was, however, a significant decrease in symptom scores during the magnesium treatment period (median difference $(95 \% \mathrm{CI}) 3.8(0.5-$ 7.0) score points per 7 days; $\mathrm{p}=0.02$ ) (fig. 1), and a reduction in bronchodilator use which was of marginal statistical significance (median difference $(95 \%$ CI) $6.0(-1-10.5)$ doses per 7 days less on magnesium; $\mathrm{p}=$ $0.051)$. There were no significant period or order effects, and there was no correlation between initial level of magnesium intake during the run-in period and change in any of the outcome variables.

Mean (SEM) serum magnesium at the beginning of the placebo and magnesium treatment periods were 0.81 (0.02) and 0.84 (0.01) mM, respectively. Serum magnesium was significantly higher at the end of the magnesium than at the end of the placebo treatment period (0.86 vs $0.80 \mathrm{mM}$, respectively; mean difference $(95 \%$ CI) $0.062(0.01-0.12) \mathrm{mM}, \mathrm{p}=0.025)$. Mean (SEM) 24 $\mathrm{h}$ urine magnesium excretion at the beginning of the placebo and magnesium treatment periods were 3.56 (0.46) and 4.64 (1.06) $\mathrm{mmol}$, and $24 \mathrm{~h}$ magnesium excretion was significantly higher at the end of the magnesium than at the end of the placebo treatment period (5.61 vs $3.88 \mathrm{mmol}$; mean difference (95\% CI) 1.73 (0.64-2.82) mmol; $\mathrm{p}=0.004)$.

Leucocyte separation took around $2 \mathrm{~h}$ to complete and, therefore, only 13 subjects who could attend the study centre early in the day were able to provide samples for this analysis. The mean (SEM) white cell magnesium levels at the beginning of placebo and magnesium treatment periods were $4.14(0.17)$ and $4.23(0.35)$ $\mu \mathrm{g} \cdot \mathrm{mg}^{-1}$ protein, respectively. There was no significant difference between the white cell magnesium levels at the end of placebo and magnesium treatment periods when compared by Student's t-test for paired data (mean difference (95\% CI) $0.69(0.13-1.51) \mu \mathrm{g} \cdot \mathrm{mg}^{-1}$ protein; $\mathrm{p}=0.86$ ). There was also no correlation between serum and white cell magnesium measurements $(\mathrm{r}=0.18)$, nor between dietary magnesium intake during the run-in week and baseline leucocyte magnesium levels $(r=0.43)$. 


\section{Discussion}

The aim of this study was to determine whether changing oral magnesium intake over a 3 week interval affected asthma control, measured in terms of airway calibre (FEV1), airway reactivity (PD20,FEV1 to methacholine), diurnal variability in peak flow (amplitude percentage mean PEF), symptom scores and bronchodilator use. As far as we are aware, this is the first study to assess the effect of alteration of magnesium intake on asthma control. Therefore, at the time of designing the study, we had no existing data on which to base decisions on design aspects, such as length of the treatment periods and wash-out period, the magnitude of any likely beneficial effect within subjects, magnitude of achievable change in magnesium intake, or ideas of likely compliance or ill effects of altering magnesium intake. These aspects of design were, therefore, determined on relatively arbitrary grounds for what is, in effect, a pilot study of magnesium effects in asthma.

To ensure subject and physician blinding it was considered most appropriate to replace magnesium in the form of tablet supplementation, rather than by dietary manipulation. The aim was to vary magnesium intake within the range of effect $\left(200-600 \mathrm{mg}^{\left.-\mathrm{day}^{-1}\right)}\right.$ on FEV1 and airway reactivity observed in our previous epidemiological study [22]. The analysis of magnesium intake from food diaries during 1 week of each treatment period showed that subjects consumed around $210 \mathrm{mg} \cdot$ day $^{-1}$ dietary magnesium during placebo and magnesium treatment periods, and confirms that subjects were compliant with the low magnesium diet, and the higher serum magnesium levels and urinary magnesium excretion at the end of the magnesium treatment period demonstrate that we were successful in our attempt to create periods of low and high magnesium intake. Although we recognize that the changes in diet we introduced may have influenced intakes of other nutrients, the use of placebo or magnesium supplements as the primary means of dietary manipulation ensures that any observed differences between the treatment limbs are likely to be due specifically to differences in magnesium intake.

At the end of the study, we observed a small but nonsignificant improvement in FEV1, a decrease in bronchodilator use of marginal statistical significance, and a significant reduction in symptom scores during the high magnesium period. There was also a nonsignificant increase in airway reactivity and a lesser improvement in diurnal variability in PEF during the magnesium period. Our primary outcome was FEV1, and since we had no within-subject estimates of magnesium effect we designed our study arbitrarily to detect a $150 \mathrm{~mL}$ difference between groups. In the event, the difference observed was $72 \mathrm{~mL}$, and a retrospective power calculation revealed that a study of approximately 88 individuals would be needed for an effect of this size to be detected as significant. Thus, if this was indeed a true magnesium effect, the present study was considerably underpowered to detect it, and it is likely that this is also true of other outcomes. Despite this negative result, however, the significant reduction in symptom scores and the borderline significant reduction in bron- chodilator use whilst asthmatic subjects were receiving a higher magnesium intake suggests that the period of magnesium supplementation during the study may have been beneficial.

The lack of significant effects on FEV1, PD20,FEV1, or amplitude percentage mean peak flow, may have occurred because the 3 week study treatment periods were too short to correct magnesium deficiency sufficiently, or to deplete an individual with adequate magnesium stores; our biochemical measurements of magnesium lend some support to this interpretation. Previous studies have suggested that serum magnesium measurements tend to reflect short-term variations in intake but not total body magnesium stores [29, 30], whilst leucocyte magnesium levels, which correlate poorly with serum magnesium measurements [23], are more closely correlated with total body stores. Our finding of a higher serum magnesium but no change in leucocyte magnesium would, therefore, suggest that our treatment periods were indeed too short to make a marked impact on total body stores. We were also unable to determine how much of the magnesium supplementation preparation was absorbed, or indeed whether a higher dose of magnesium would have been more effective. Censoring of PD20,FEV1 values on three occasions in two subjects is likely to have contributed to a minor degree of underestimation of the effect on PD20,FEV1 but would not explain the qualitative results.

It is also possible that any effect of increasing dietary magnesium intake is dependent on initial level of intake, though we looked for and found no correlation between improvement in any of the outcome measures during the magnesium treatment period of our study and initial magnesium intake measured during the runin period. It is also possible that increased magnesium intake may be more beneficial to asthmatic subjects with more severe disease than those included in this study, who had a mean FEV1 of $78 \%$ pred, and were largely maintained on low-dose inhaled corticosteroids and beta-agonist therapy as required. It has been suggested that magnesium may potentiate the effect of beta-adrenoceptor agonists [31] and, thus, there may be an important interaction between asthma treatment and the effect of magnesium, which may be relevant to this study.

Despite finding no significant effect on measures of airflow or airway reactivity, the demonstration of a significant improvement in symptom scores, and a reduction in bronchodilator use of borderline significance suggest some degree of benefit during magnesium supplementation. This inference is tempered by the increase in airway reactivity, which was also of borderline significance, though it is possible that the decreased use of bronchodilator during the study, which was equivalent to just under 1 dose day $^{-1}$, may have contributed to this change. The decreased use of bronchodilator is certainly likely to have increased PEF variability during the magnesium treatment period, and, to a lesser extent, reduced FEV1.

These preliminary findings, therefore, suggest that a simple intervention involving magnesium supplementation may have induced an overall improvement in symptomatic control of asthma. On the basis of these findings and in view of the relative simplicity of this 
intervention either by means of supplementation or dietary change, a more extended study of the effect of a high magnesium intake sustained over a longer period on asthma control is now indicated.

Acknowledgements: N. Lawson, Consultant Clinical Chemist, provided helpful advice during the planning stages of this study. K. Richards helped with recruitment of asthmatic subjects, and S. Pacey and $\mathrm{H}$. Tattersall in the Pharmacy Department of the City Hospital provided randomization codes and placebo supplementation tablets.

\section{References}

1. Altura BM, Altura BT, Carella A. Magnesium deficiencyinduced spasms of umbilical vessels: relation to preeclampsia, hypertension, growth retardation. Science 1983; 221: 376-378

2. Spivey WH, Skobeloff EM, Levin RM. Effect of magnesium chloride on rabbit bronchial smooth muscle. Ann Emerg Med 1990; 19: 1107-1112.

3. Gilbert AJ. Microscopic observations of pulmonary artery reactions. J Pharm Exp Ther 1938; 62: 228-235.

4. Del Castillo J, Engbaek L. The nature of the neuromuscular block produced by magnesium. J Physiol 1954; 124: 370-384.

5. Bois P. Effect of magnesium deficiency on mast cells and urinary histamine in rats. Br J Exp Path 1963; 44: 151-155.

6. Kemp PA, Gardiner SM, March JE, Bennett T, Rubin $\mathrm{PC}$. Effects of $\mathrm{NG}^{\mathrm{G}}$-nitro-L-arginine methyl ester on regional haemodynamic responses to $\mathrm{MgSO}_{4}$ in conscious rats. Br J Pharmacol 1994; 111: 325-331.

7. Nadler JL, Goodson S, Rude RK. Evidence that prostacyclin mediates the vascular action of magnesium in humans. Hypertension 1987; 9: 379-383.

8. Bloch H, Silverman R, Mancherje N, Grant S, Jagminas $\mathrm{L}$, Scharf SM. Intravenous magnesium sulfate as an adjunct in the treatment of acute asthma. Chest 1995; 107: 1576-1581.

9. Noppen M, Vanmaele L, Impens N, Schandevyl W. Bronchodilating effect of intravenous magnesium sulphate in acute severe bronchial asthma. Chest 1990; 97: 373-376.

10. Okayama H, Aikawa T, Okayama M, Sesaki H, Mue S, Takishima T. Bronchodilating effect of intravenous magnesium sulphate in bronchial asthma. J Am Med Assoc 1987; 257: 1076-1078.

11. Rolla G, Bucca C, Caria E, et al. Acute effect of intravenous magnesium sulphate on airway obstruction of asthmatic patients. Ann Allergy 1988; 61: 388-391.

12. Skobeloff EM, Spivey WH, McNamara RM, Greenspon L. Intravenous magnesium sulphate for the treatment of acute asthma in the emergency department. $J$ Am Med Assoc 1989; 262: 1210-1213.

13. Brunner EH, Delabroise AM, Haddad ZH. Effect of parenteral magnesium on pulmonary function, plasma cAMP, and histamine in bronchial asthma. J Asthma 1985; 22: 3-11.
14. Plaisance P, Hibon A, Adnet F, Bouexiere D, Richard C, Payen B. Potentiation of beta ${ }_{2}$-agonists by inhaled magnesium sulfate in prehospital management of acute bronchial asthma: a double-blind study (Abstract). Am J Respir Crit Care Med 1994; 149: A190.

15. Rolla G, Bucca C, Arossa W, Bugiani M. Magnesium attenuates methacholine-induced bronchoconstriction in asthmatics. Magnesium 1987; 6: 201-204.

16. Rolla G, Bucca C, Bugiani M, Arossa W, Spinaci S. Reduction of histamine-induced bronchoconstriction by magnesium in asthmatic subjects. Allergy 1987; 42: 186-188.

17. Bernstein WK, Khastgir T, Khastgir A, et al. Lack of effectiveness of magnesium in chronic stable asthma. Arch Intern Med 1995; 155: 271-276.

18. Tiffany BR, Berk WA, Todd IK, White SR. Magnesium bolus or infusion fails to improve expiratory flow in acute asthma exacerbations. Chest 1993; 104: 831-834.

19. Green SM, Rothrock SG. Intravenous magnesium for acute asthma: failure to decrease emergency treatment duration or need for hospitalisation. Ann Emerg Med 1992; 21: 260-265.

20. Matusiewicz S, Cusack S, Greening AP, Crompton GK. A double-blind, placebo-controlled, parallel group study of intravenous magnesium sulphate in acute severe asthma (Abstract). Eur Respir J 1994; 7 (Suppl. 18): 14s.

21. Chande VT, Skoner DP. A trial of nebulised magnesium sulphate to reverse bronchospasm in asthmatic patients. Ann Emerg Med 1992; 21: 1111-1115.

22. Britton J, Pavord I, Richards K, et al. Dietary magnesium, lung function, wheezing and airway hyperreactivity in a random adult population sample. Lancet 1994; 344: 357-362.

23. Elin RJ, Hosseini JM. Magnesium content of mononuclear blood cells. Clin Chem 1985; 31(3): 377-380.

24. Yan K, Salome C, Woolcock AJ. Rapid method for measurement of bronchial responsiveness. Thorax 1983; 38: 760-765.

25. Britton J, Mortagy A, Tattersfield A. Histamine challenge testing: comparison of three methods. Thorax 1986; 41: $128-132$.

26. Hills M, Armitage P. The two-period, cross-over clinical trial. Br J Clin Pharmacol 1979; 8: 7-20.

27. Tweeddale PM, Alexander F, McHardy GJR. Shortterm variability in FEV1 and bronchodilator responsiveness in patients with obstructive ventilatory defects. Thorax 1987; 42: 487-490.

28. Becklake MR, Permutt S. Evaluation of tests of lung function for screening for early detection of chronic obstructive lung disease: In: Macklem PT, Permutt S, eds. The Lung in the Transition Between Health and Disease. New York, Marcel Dekker, 1979; pp. 345-387.

29. Opie LH, Hunt BG, Finlay JM. Massive small bowel resection with malabsorption and negative magnesium balance. Gastroenterology 1964; 47: 415-420.

30. Alfrey AC, Miller NL, Butkus D. Evaluation of body magnesium stores. J Lab Clin Med 1994; 84: 153-162.

31. Skorodin MS, Freebeck PC, Yetter B, Nelson JE, Van de Graaff M, Walsh JM. Magnesium sulphate potentiates several cardiovascular and metabolic actions of terbutaline. Chest 1994; 105: 701-705. 